\title{
RATIONALE BEHIND THE COMPARISON OF KABA SURAM AND OTHER SURAMS WITH CORONAVIRUS DISEASE AND OTHER VIRAL FEVERS - A LITERATURE REVIEW
}

\author{
MANICKAVASAGAM RENGARAJU ${ }^{1 *}$, KANAKAVALLI KADARKARAI $^{2}$ \\ ${ }^{1}$ Siddha Clinical Research Unit, Safdarjung Hospital Campus, New Delhi. (A unit under Central Council for Research in Siddha, Min. of \\ AYUSH, Govt. of India), India, ${ }^{2}$ The Director General, Central Council for Research in Siddha, Min. of AYUSH, Govt. of India, Chennai, Tamil \\ Nadu, India. Email: nismanick@gmail.com
}

Received: 22 July 2021, Revised and Accepted: 26 August 2021

\section{ABSTRACT}

Objectives: This article intended to discuss about the rationale behind the comparison of coronavirus disease (COVID 19) and other viral fevers signs and symptoms with "Kaba Suram" and others Surams signs and symptoms with available source of Siddha literatures. Materials and Methods: Research Type: Systemic review. Design: Literature review will be done by collection of available text references from authenticated Siddha texts. Reference Authenticated Siddha Texts: 1. Yugimuni Vaithya Sinthamani, 2. Sura Vagadam, 3. Theran Karisal, 4. Theran Yamaga Venba, and 5. Sura Venba. Conclusion: Based on inference derived from Results and Discussion, we came to conclude that signs and symptoms of various viral fevers (COVID-19, dengue, chikungunya, and swine flu) are most commonly matched with the signs and symptoms of Kaba Suram, Pitha Suram, Vatha Suram, and Kaba Pitha Suram which are already mentioned in Siddhar authenticated texts.

Keywords: Chikungunya, Coronavirus disease, Dengue, Siddha, Suram, Swine Flu.

(c) 2021 The Authors. Published by Innovare Academic Sciences Pvt Ltd. This is an open access article under the CC BY license (http://creativecommons.org/ licenses/by/4.0/) DOI: http://dx.doi.org/10.22159/ijas.2021v9i5.42835. Journal homepage: https://innovareacademics.in/journals/index.php/ijas

\section{INTRODUCTION}

Siddha system is one of the traditional medical systems of India which is most popular in Southern Peninsula of India [1-3]. Coronavirus disease (COVID-19) is one of the viral infections which is a dangerous infection producing sudden respiratory arrest and leads maximum up to death. This infection is caused by a variety of RNA group of virus, that is, severe acute respiratory syndromeCoV-2. Like COVID-19, chikungunya, dengue, and swine flu are the viral infections causing causalities as pandemic in the past years throughout the world [4]. These are the major threatening viral fevers to the world community not only in developing countries but also in developed countries. The similarity between all these viruses is all four belong to RNA group of viruses [5]. Health-care communities are trying to find out the apt treatment for these viral infections. Role of traditional medicines throughout the world is notable in the management of all these fevers. Specifically, Indian traditional systems (AYUSH) are contributing lot to community in treatment/prophylactic aspects of all these fevers. Siddha system of medicine also playing an essential role in treatment and management aspects of these viral fevers. In general, "fever" is mentioned in Siddha Text as "Suram," Kaichal," Veppu," Kanal," and "Vedhuppu." [6] There are 64 types of fever classified in Siddhar texts based on causative factors, signs and symptoms, and imbalance of Thirithodam (three humor Vatha-Pitha-Kapham) [7]. This article intended to discuss about the rationale behind the comparison of COVID 19 and other viral fevers signs and symptoms with "Kaba Suram" and others Surams signs and symptoms with available source of Siddha literatures.

Siddha system and pandemics/epidemics

In Siddha system, epidemics/pandemics are mentioned as "Uzhi Noi" or "Kothari Noi" [8]. In general, they are classified under "Kollai Noikal" which are most commonly occurred the time of "Ayana Santhi" [9] months (means end months of Uthara Ayanam and Thatchana Ayanam), fall on Adi and Margazhi month in Tamil Calendar [10]. It is believed that in those days, immunity of human being will be low based on Thirithoda Theory [11,12].
In this context, the signs and symptoms mentioned under "Kaba Suram" and some other Surams can be compared with the signs and symptoms of the COVID-19 and other viral fevers [6,12].

Viral fevers comparison with various types of Suram as per Siddha literatures

\begin{tabular}{lll}
\hline S. No. & Name of the viral fevers & $\begin{array}{l}\text { Suitable type of Suram as per } \\
\text { Siddha literatures }\end{array}$ \\
\hline 1 & COVID-19 & Kaba Suram \\
2 & Dengue & Pitha Suram \\
3 & Chikungunya & Vatha Suram \\
4 & Swine Flu & Kaba Pitha Suram \\
\hline
\end{tabular}

\section{MATERIALS AND METHODS}

It is a systemic review done by collection of available text references from authenticated Siddha texts. The following authenticated texts were taken as source of references.

1. Yugimuni Vaithya Sinthamani [13], 2. Theran Karisal [14], and 3. Theran Yamaga Venba [15].

\section{RESULTS AND DISCUSSION}

The following Table 1 comparing with the signs and symptoms of COVID-19 declared by the WHO [16] with signs and Symptoms of Kaba Suram mentioned various Siddha authenticated literatures [6,7].

Based on above signs and symptoms of COVID-19 classified by the WHO with Kapha Suram which are mentioned in Siddha literatures, it is found that $95 \%$ (excluding headache) of major and $90 \%$ (excluding loss of smell and red eyes) of minor signs and symptoms matched with the WHO classification. Overall, 14 out of 17 signs and symptoms classified by the WHO classification of COVID-19 matched with Kapha Suram which are mentioned in Siddha authenticated literature.

The following Table 2 comparing with the signs and symptoms of dengue declared by the WHO [17] with signs and symptoms of Pitha Suram mentioned in various Siddha authenticated literatures $[4,5]$. 
Table 1: COVID-19 versus Kaba Suram

\begin{tabular}{|c|c|c|c|}
\hline S. No. & $\begin{array}{l}\text { WHO classified signs } \\
\text { and symptoms }\end{array}$ & Siddha terminology & Siddha quotes mentioned in Siddha texts with reference \\
\hline 1 & Fever & Suram, Kaichal, Veppu & 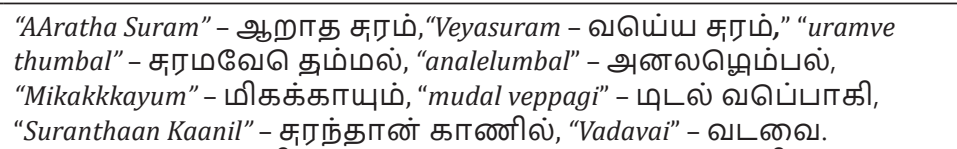 \\
\hline 2 & Cough & Irumal & $\begin{array}{l}\text { "AAa yirumalu" - ஆயிரிமலு, "Irumba" - இர,ம்ப “nirumal” - நிர,மல், “Maar } \\
\text { nothalirumal lilaippu" - மார்நோதல் இருமல் இளப்பு }\end{array}$ \\
\hline 3 & Fatigue/tiredness & $\begin{array}{l}\text { Balaheenam, Asathi, } \\
\text { Kalaippu }\end{array}$ & "Masathyum" - மசதியும் \\
\hline 4 & Loss of taste/loss of smell & Arusi, Suvaiyinmai & $\begin{array}{l}\text { "Naaku munnuna uvurusi yillai" - உர,சியில்ல, } \\
\text { "Urusiyillai" - உரசியில்லன }\end{array}$ \\
\hline 5 & Sore throat & Thondaikattu & $\begin{array}{l}\text { "Maar Thondai Nokum" - மார் தொண்டந நோகம் , “Sinthaba mitaru } \\
\text { nonthu” - }\end{array}$ \\
\hline 6 & Aches and pain & Vali,Ulaivu & “Mulaivu” - உளவை, “Udambu konthazhatrum” - உடல் உளயைம் \\
\hline 7 & $\begin{array}{l}\text { Shortness of breathing/ } \\
\text { difficulty in breathing }\end{array}$ & Illaippu,Meel Moochu & $\begin{array}{l}\text { "Ayasa mersu vaasam" - ஆயாச மேற் சுவாசம் } \\
\text { "Moochilaithal" - மூச்சிளத்தல் } \\
\text { "Lilaipuntagum" - லிளரப்புண்டாகம் } \\
\text { "Maar nothalirumal lilaippu" - மார் நமாதல் இரமல் இளபைப்பு, "Men } \\
\text { moochathal" - மேல் ஜூச்சாதல் }\end{array}$ \\
\hline 8 & Mental confusion & Manakulappam,Thayakam & $\begin{array}{l}\text { "Thiyakkamural” - தியக்கமுறல் “Manathuvalum” - மனந்தவள,ம் } \\
\text { "Thiyankituthal” - தியங்குதல் }\end{array}$ \\
\hline 9 & Diarrhea & Kalichal, Athisaram & $\begin{array}{l}\text { "Kurukutha lathisara" - கறக்குறத்த லதிசார, "mudalil malasalanthaan } \\
\text { nuraikil" - உடலில் மலசல நரகைகில், "Vayiru mudan kaliyu" - உடலில் } \\
\text { மலசல நரகைகில் }\end{array}$ \\
\hline 10 & $\begin{array}{l}\text { Discoloration of body } \\
\text { parts (face, fingers, and } \\
\text { toes) }\end{array}$ & Veluupu, Paandu & $\begin{array}{l}\text { “Ayira Nayananmei Yanaiyi nirame” - ஆயிரநாயனன்மை யானயைி } \\
\text { நிறமே } \\
\text { “Seiya mukank karampaatham velluthuk kaatal” - முகம் கரம் பாதம் } \\
\text { வளெ,த்தக்காட்டல் } \\
\text { “Mukamathu vellukum" - முகமத வளெக்கும் } \\
\text { “Naaku Mukam Velluthu Kaanaal” - நாக்க முகம் வளெத்தக்காணல் }\end{array}$ \\
\hline 11 & Loss of speech & Pechinmai & “Vaayu laral” - வாய் உளறல் \\
\hline 12 & Eye symptoms & Kan Noigal & "Peelai vazhiyu mirukannum" - \\
\hline 13 & Skin rashes & Thol Arippu, Thinavu & $\begin{array}{l}\text { "Udal Thinav" - உடல் தினவு } \\
\text {, Thinaveduthal - தினவடெத்தல் }\end{array}$ \\
\hline 14 & Chest pain & Marbu vali, Nenjulaithal & $\begin{array}{l}\text { "Maar Thondai Nokum" - மார்ததொண்டநைோகும் } \\
\text { "Maar nothalirumal lilaippu" - மார்நதோதல் இருமலிளப்பு }\end{array}$ \\
\hline
\end{tabular}

(Headache, loss of smell, and red eyes are the symptoms which are not matching with Siddha literature)

Table 2: Dengue versus Pitha Suram

\begin{tabular}{|c|c|c|c|}
\hline S. No. & $\begin{array}{l}\text { WHO classified signs } \\
\text { and symptoms }\end{array}$ & Equivalent Siddha terminology & Siddha quotes mentioned in Siddha texts with reference \\
\hline 1 & High fever & Suram, Kaichal, Veppu & “Itaivitatha Suram" - இடைவிடாத சுரம் \\
\hline 2 & Severe headache & Thalai Sutral, Thalai Kanathal & "Thalai Sutral" - தலைச்சுற்றல் \\
\hline 3 & Rapid breathing & Ilaippu & "Ilaippu" - இளைப்பு, "Mel Moochu Iraithal” - மேல் மூச்சு இரதைல் \\
\hline 4 & Headache & $\begin{array}{l}\text { Thalai Vali, Thalai Kanathal, } \\
\text { Thalai Noi }\end{array}$ & $\begin{array}{l}\text { "Thalai noi” - தலலைய், "Thalai Kanathal” - தலை கனத்தல், } \\
\text { "Thalai kutainthu kutainthu Nothal" - தலை கடநை்த கடநைத } \\
\text { ோதல் }\end{array}$ \\
\hline 6 & Nausea & Velokalam & “Okkalam” - ஓக்காளம் \\
\hline 7 & Vomiting & Vanthi, Sathi & “Vanthi” - வாந்தி \\
\hline 8 & Diarrhea & Kalichal, Athisaram & Vayiru Kalithal - வயிற கழிதல் \\
\hline 9 & Chills & Kulir & “Udal Nadunkum" - உடல் நடுங்கம், “Udal Kulirchi” - உடல் களிர்ச்சி \\
\hline 10 & Skin rashes & Thinavu, Thimir & $\begin{array}{l}\text { "Udal Neruppu Pol Erithal” - உடல் நெரப்பு ோல் எரிதல், "Udal } \\
\text { Punnathal" - உடல் புண்ணாதல் }\end{array}$ \\
\hline 11 & Severe abdominal pain & Vayiru Vali & "Vayiroothi Ulaithal" - வயிறததி உளதைல் \\
\hline 13 & Fatigue & Kalaippu & $\begin{array}{l}\text { "Kalaipu” - களைப்பு, "Udal Vanmai Kuraithal" - உடல் வன்மை } \\
\text { கறதைல் }\end{array}$ \\
\hline 14 & Anorexia & Anna veruppu, Pasiyinmai & Anna veruppu - அன்ன வெறப்பு \\
\hline 15 & $\begin{array}{l}\text { Restlessness and other } \\
\text { psychological problems }\end{array}$ & Mana Ulaichal and Amaithinmai & $\begin{array}{l}\text { "Mirantu Parthal" - மிரண்டு பார்த்தல், "Mananilai maruthal" - } \\
\text { மனநிலன மாறதல், "Mana kalakkam" - மனக்கலக்கம், Ullam } \\
\text { Kothithal - உள்ளம் ொதித்தல், Vai Pithatral - வாய் பிதற்றல், } \\
\text { "Puthi Mayankal" - புத்தி மயங்கல், "Thavithal" - தவித்தல் }\end{array}$ \\
\hline
\end{tabular}

(Joint pain or muscle pain and blood vomiting are the symptoms which are not matching with Siddha literature) 
Based on above signs and symptoms of dengue fever classified by the WHO with signs and symptoms of Pitha Suram which are mentioned in Siddha literatures, It is found that $95 \%$ (joint or muscle pain) of major signs and symptoms and 95\% (excluding blood vomiting) of minor signs and symptoms matched with the WHO classification. Overall, 15 out of 17 signs and symptoms classified by the WHO classification of dengue fever matched with signs and symptoms of Pitha Suram which are mentioned in Siddha authenticated literature.

The following Table 3 comparing with the signs and symptoms of chikungunya declared by the WHO [18] with signs and symptoms of Vatha Suram mentioned various Siddha authenticated literatures [4,5].

Based on above signs and symptoms of chikungunya classified by the WHO with signs and symptoms of Vatha Suram which are mentioned in Siddha literatures, it is found that 95\% (excluding oral ulcers) of major signs and symptoms and 95\% (excluding swelling) of minor signs and symptoms matched with the WHO classification. Overall, 12 out of 14 signs and symptoms classified by the WHO classification of chikungunya matched with signs and symptoms of Vatha Suram which are mentioned in Siddha authenticated literature.

The following Table 4 comparing with the signs and symptoms of swine flu declared by WHO [19] with signs and symptoms of Kaba Pitha Suram mentioned various Siddha authenticated literatures $[4,5]$.

Based on above signs and symptoms of swine flu classified by the WHO with signs and symptoms of Kapha Pitha Suram which are mentioned in Siddha literatures, it is found that $100 \%$ of major signs and symptoms matched with the WHO classification and $100 \%$ of minor signs and symptoms matched with the WHO classification. Overall, 13 out of 13 signs and symptoms classified by the WHO classification of swine flu matched with signs and symptoms of Kapha Pitha Suram which are mentioned in Siddha authenticated literature.

Table 3: Chikungunya versus Vatha Suram

\begin{tabular}{|c|c|c|c|}
\hline S. No. & $\begin{array}{l}\text { WHO classified signs } \\
\text { and symptoms }\end{array}$ & Equivalent Siddha terminology & Siddha quotes mentioned in Siddha texts with reference \\
\hline 1 & Fever & Suram, Kaichal, Veppu & “Suramathu Mikunthu Kayum” - சுரமத மிகுந்த காயும் \\
\hline 2 & Arthralgia & Mootu Vali,Santhu Vali ,Keel Vayu & $\begin{array}{l}\text { "Kai kal Kaduppu” - கைகால் கடுப்பு, "Poruthu thorum mulaivakum" } \\
\text { - ொரத்த வாறம் மளவைாகம், "Kai kaalkal valithaal" - } \\
\text { கைகால்கள் வலித்தல், "Puranthal veluthu veenki pothal" - புறந்தாள் } \\
\text { வளெத்த வீங்கிப்கேதல், "Santhukal valaal aruppathu Pol Valithal" } \\
\text { - சந்தங்கள் வாளால் அறப்பத ோல் வலித்தல், "Pootukalil Vali" - } \\
\text { பூட்டகளில் வலி }\end{array}$ \\
\hline 3 & Backache & Iduppulaivu & "Iduppu kutainthu Nothal" - இடுப்பு கடைந்த ோதல் \\
\hline 4 & Headache & $\begin{array}{l}\text { Thalai Vali, Thalai Kanathal, } \\
\text { Thalai Noi }\end{array}$ & $\begin{array}{l}\text { "Thalai noi" - தலைடேய், "Thalai Kanathal" - தடை கனத்தல், "Thalai } \\
\text { kutainthu kutainthu Nothal" - தலை கடைந்த கடைந்த ோதல் }\end{array}$ \\
\hline 5 & Retro - Orbital Pain & Kan Vali & "Kan Vali" - கண் வலி \\
\hline 6 & Vomiting & Vanthi, Vellokalam, Sathi & "Vanthi” - வாந்தி \\
\hline 7 & Diarrhea & Kalichal, Athisaram & "Kalithal” - கழிதல் \\
\hline 8 & Chills & Kulir & "Udal Kulir" - உடல் களிர் \\
\hline 9 & $\begin{array}{l}\text { Skin problems } \\
\text { 1. Exfoliative dermatitis, } \\
\text { 2. Hyperpigmentation, } \\
\text { 3. Skin rashes }\end{array}$ & $\begin{array}{l}\text { Thol kurigunankal } \\
\text { 1.Thol Vedippu,2. Thol Karu } \\
\text { niramadaithal 3. Thinavu, Thimir }\end{array}$ & $\begin{array}{l}\text { 1. "Thol surunkum" - ோல் சுரங்கம், } 2 . \text { "Udal Muluvathm } \\
\text { Karuniramadaithale" - உடல் மழவதம் கரநநிறமடைதல், "Udal } \\
\text { muluvathum karuthu vali" - உடல் முவவதம் கரதத் வலி, 3. "Thimir" - } \\
\text { திமிர் }\end{array}$ \\
\hline
\end{tabular}

(Stomatitis and swelling are the symptoms which are not matching with Siddha literature)

Table 4: Swine flu versus Kaba Pitha Suram

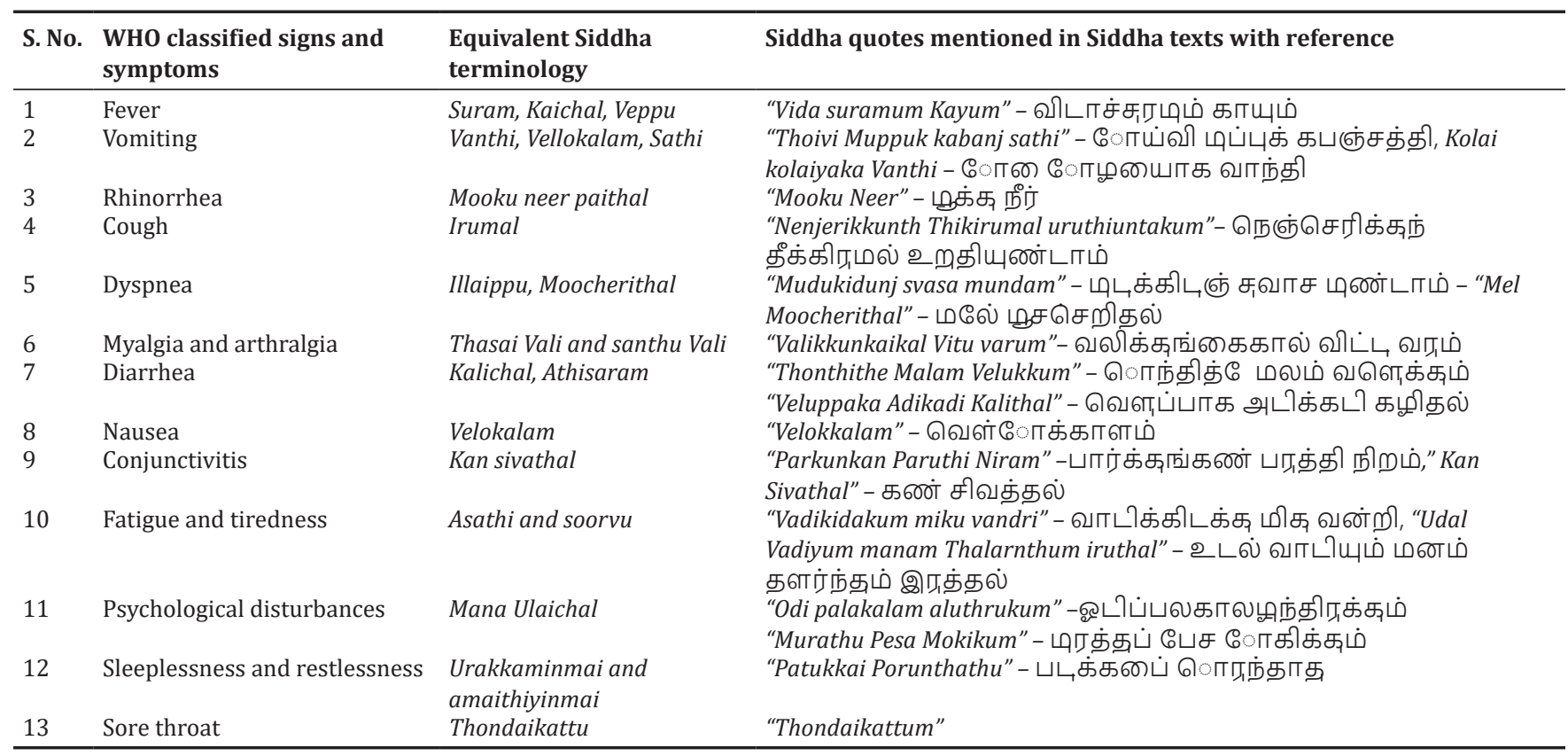




\section{CONCLUSION}

Based on inference derived from results and discussion, we came to conclude that signs and symptoms of various viral fevers (COVID-19, dengue, chikungunya, and swine flu) are most commonly matched with the signs and symptoms of Kaba Suram, Pitha Suram, Vatha Suram, and Kaba Pitha Suram which are already mentioned in Siddhar authenticated texts.

Like classification of 64 fevers, separate treatment of regimen for individual fevers also mentioned in Siddhar texts. Hence, based on this comparison, the medicines which are mentioned for the Kaba suram, Pitha suram, Vatha Suram, and Kaba Pitha Suram will useful in the management of COVID-19, dengue, chikungunya, and swine flu.

Note: Various RCT with Kaba Sura Kudineer - initiated and carried out by CCRS for the management of COVID 19 under the guidance of Min. of AYUSH, Govt. of India. (Multicentric Prophylactic Population Study, Stanley Medical College Siddha Study [20], Theni Medical College Siddha Study, SRM Siddha Study, Vyasarbadi Study, and GIMS Noida Siddha Study [21]).

\section{AUTHORS' CONTRIBUTIONS}

MR conceived and developed the concept of this paper. MR also wrote and drafted the whole manuscript. KK helped in the finalization of the manuscript.

\section{CONFLICTS OF INTEREST}

Authors declared that there are no conflicts of interest in this stage.

\section{REFERENCES}

1. The Editors of Encyclopedia Britannica. Siddha Medicine, Encyclopedia Britannica; 2018. Available from: https:/www.britannica.com/science/ Siddha-medicine. [Last accessed on 2021 Aug05].

2. Manickavasagam R. Siddha literature repositories in and outside Tamil Nadu. Eur J Biomed Pharm Sci 2017;4:195-7.

3. Manickavasagam R, Rajam S, Shanthi DM. Pura Maruthuva Muraigal (External therapies and methods) in siddha system-a review. World J Pharm Pharm Sci 2017;6:523-33.

4. Huremović D. Brief history of pandemics (pandemics throughout history). Psychiatry Pandemics 2019;2:7-35.

5. Baveja CP. Text Book of Micro-biology. $6^{\text {th }}$ ed. New Delhi: Arya Publications; 2018. p. 20-104.
6. Kuppusamy M. Maruthuvam. $3^{\text {rd }}$ ed. Chennai: Department of Indian Medicine and Homoeopathy; 1967. p. 2-78.

7. Shanmugvelu N. Noi Nadal Noi Mudal Nadal. Part II Chennai: Department of Indian Medicine and Homoeopathy; 1967. p. 224.

8. Sambasivam Pillai TV. Dictionary of Medicine, Chemistry, Botany and Allied Science. Vol. 2. Chennai: Directorate of Indian Medicine and Homeopathy; 1991. p. 1724.

9. Rajan D. Noi Illa Neri. Chennai: Directorate of Indian Medicine and Homeopathy; 2016. p. 223.

10. Ministry of AYUSH, Government of India; 2020. Available from: https://www.ayush.gov.in/docs/siddha-guidelines.pdf. [Last accessed on 2021 Aug 05].

11. Shanmugvelu N. Noi Nadal Noi Mudal Nadal. Part I. Chennai: Department of Indian Medicine and Homoeopathy; 1967. p. 184.

12. Manickavasagam R, Kanakavalli K. History of siddha and epidemics/ pandemics-a review. Int J AYUSH 2021;10:52-7.

13. Ramachandran P. Yugi Vaithiya Sinthamani 800. $3^{\text {rd }}$ ed. Chennai: Thamarai Noolagam; 2018. p. 22-9.

14. Ramachandran P. Therayar Karisal 300, Therayar. $1^{\text {st }}$ ed. Chennai: Thamarai Noolagam; 1915. p. 44-6.

15. Therayar Y. Therayar Yamagam. $2^{\text {nd }}$ ed. Chennai: Thamarai Noolagam; 2003. p. 78-9.

16. The Editors and Website of World Health Organization. Available from: https://www.who.int/health-topics/coronavirus tab=tab_1. [Last accessed on 2021 Aug 05].

17. The Editors and Website of World Health Organization. Available from: https://www.who.int/tdr/publications/documents/dengue-diagnosis. pdf. [Last accessed on 2021 Aug05].

18. The Editors and website of DHS, Kerala Government. Available from: https://www.dhs.kerala.gov.in/wp-content/uploads/2020/06/ cgn_11092018.pdf. [Last accessed on 2021 Aug05].

19. The Editors and Website of World Health Organization. Available form: https://www.who.int/emergencies/diseases/novel-coronavirus-2019/ question-and-answers-hub/q-a-detail/coronavirus-disease-covid19-similarities-and-differences-with-influenza. [Last accessed on 2021 Aug 05]

20. Natarajan S, Anbarasi C, Sathiyarajeswaranc P, Manickam P, Geetha S, Kathiravan R, et al. The efficacy of siddha medicine, Kabasura Kudineer (KSK) compared to Vitamin C and Zinc (CZ) supplementation in the management of asymptomatic COVID-19 cases: A structured summary of a study protocol for a randomized controlled trial. Trials 2020;21:892.

21. Anurag S, Rengaraju M, Srivastava S, Narayan V, Gupta V, Upadhayay R. A double blinded placebo controlled comparative clinical trial to evaluate the effectiveness of Siddha medicines, Kaba Sura Kudineer (KSK) and Nilavembu Kudineer (NVK) along with standard Allopathy treatment in the management of symptomatic COVID 19 patients-a structured summary of a study protocol for a randomized controlled trial. BMC J Trials 2021;22:130. 